\title{
Schlauchmagen-OP verbessert metabolisches Syndrom
}

Fragestellung: Inwieweit beeinflusst eine SchlauchmagenOperation(-OP) das Körpergewicht, den Glukose- und Lipidstoffwechsel und die Prävalenz eines metabolischen Syndroms über zwei Jahre?

Hintergrund: Adipositas $\left(\mathrm{BMI}>30 \mathrm{~kg} / \mathrm{m}^{2}\right)$ ist häufig mit Fettstoffwechselstörungen, Diabetes, Hypertonie, metabolischem Syndrom, Herz-Kreislauf-Erkrankungen und Schlafapnoe assoziiert. Das Übergewicht kann mit einer Schlauchmagen-OP, welche die Resektion der großen Magenkurvatur beinhaltet [1], deutlich reduziert werden. Jedoch gab es bis dato noch keine Langzeit-Studien, die untersucht haben, wie sich eine Schlauchmagen-OP auf das Gewicht, den Glukosestoffwechsel, die Lipidprofile und das metabolische Syndrom langfristig auswirkt.

Patienten und Methodik: Die Autoren maßen bei 52 deutlich adipösen Patienten (im Mittel 43 Jahre alt, 20 Männer) den BMI, das Gesamt-Cholesterol, die Triglyzeride, das HDL- und LDLCholesterol, Lipoprotein(a), die Glukose, den $\mathrm{HbA}_{1 \mathrm{c}}$, das Insulin und Parameter des metabolischen Syndroms einschließlich des Blutdrucks vor der OP und 6 Monate $(n=52)$ sowie $12(n=41)$ und 24 Monate $(n=5)$ danach. Ein metabolisches Syndrom (3 oder mehr der Kriterien: Abdominale Adipositas, Hypertriglyzeridämie, niedriges HDL-C, Hypertonie, antihypertensive Therapie, erhöhter Nüchtern-Blutzucker, Diabetes) hatten $42 \mathrm{~Pa}$ tienten vor der OP. Die Daten wurden retrospektiv erhoben.

Ergebnisse: Die Patienten hatten anfangs alle eine zentrale Adipositas und zu 88\% eine arterielle Hypertonie. Der BMI fiel 6 Monate nach der OP von 51 auf $40 \mathrm{~kg} / \mathrm{m}^{2}$, nach 12 Monaten auf 39 und nach 24 Monaten auf $38 \mathrm{~kg} / \mathrm{m}^{2}$ ab (Abb. 1). Die Prävalenz des me-

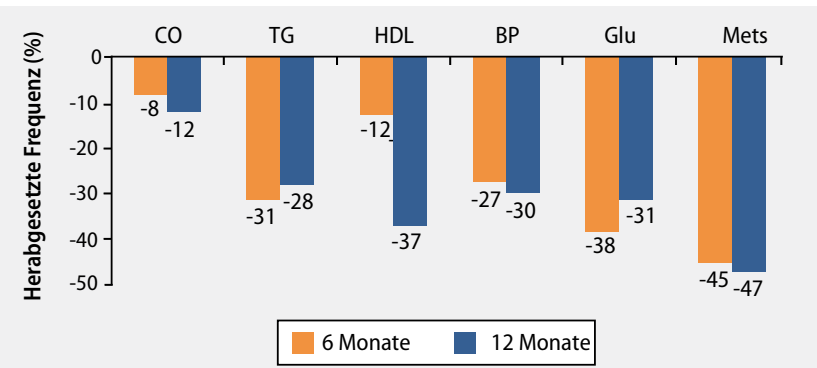

1 Prozentuale Abnahme der Prävalenz von individuellen Kriterien, die das metabolische Syndrom definieren, gemessen jeweils 6 und 12 Monate nach Schlauchmagen-OP. CO: Zentrale Adipositas, TG: Triglyzeride, HDL-Cholesterol, BP: Blutdruck, Glu: Nüchtern-Blutzucker, Mets:Metabolisches Syndrom.

tabolischen Syndroms nahm nach 6 bzw. 12 Monaten von 81 auf $36 \%$ bzw. 34\% ab. Zeitgleich sanken die Triglyzeride deutlich um 31 und $28 \%$. Ebenso eindeutig verbesserten sich die anderen Kriterien des metabolischen Syndroms: der Blutdruck sank um 27 und 30\% und der Nüchternglukosewert um 38 und 31\% (Abb. 1).

Schlussfolgerung: Aus diesen Ergebnissen schlussfolgern die Autoren, dass eine Schlauchmagen-OP die Faktoren des metabolischen Syndroms positiv beeinflussen kann - ähnlich effektiv wie die malabsorptive bariatrische Chirurgie diese Kriterien verbessert.
To VT, Hüttl TP, Lang R, Piotrowski K, Parhofer KG. Changes in body weight, glucose homeostasis, lipid profiles, and metabolic syndrome after restrictive bariatric surgery. Exp Clin Endocrinol Diabetes. 2012;120(9):547-52.

\section{-Kommentar von Prof. Dr. med. Emanuel Fritschka}

\section{Noch ein Pluspunkt dieses Verfahrens: Vitamin-Mangel unwahrscheinlich}

Erstmals wurde in der hier vorgestellten Studie untersucht, inwieweit eine Schlauchmagen-OP bei deutschen Patienten die metabolischen Parameter beeinflusst. Die Ergebnisse sind ähnlich denen der malabsorptiven bariatrischen OP-Technik. Signifikant waren die meisten Ergebnisse bereits sechs Monate postoperativ, nämlich dann, als die Gewichtsabnahme am deutlichsten war. Allerdings sind noch weitere Studien erforderlich, um den Langzeitnutzen der Schlauchmagen-OP ermessen zu können. Das Risiko eines Mangels an Thiamin, Vitamin-B6, -B12, und -D, Kalzium, Eisen, Kupfer oder Zink - wie er nach malabsorptiven bariatrisch-chirurgischen Verfahren beobachtet wurde - dürfte bei der Technik der Schlauchmagen-OP jedenfalls kein Diskussionspunkt sein.

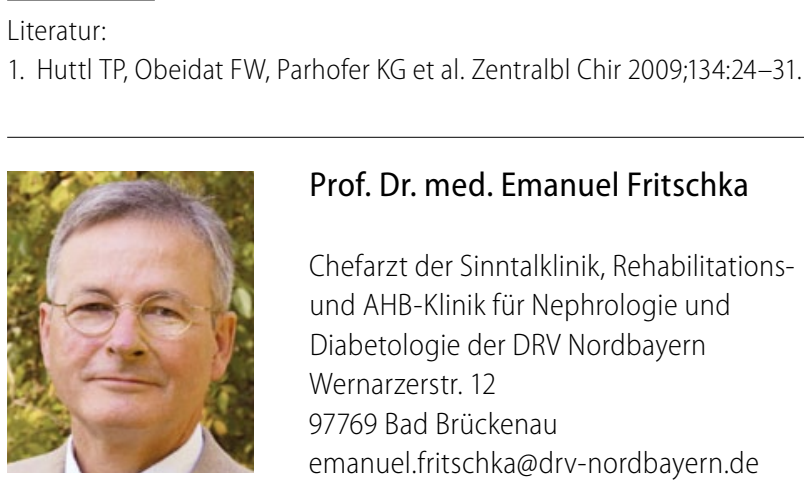

Literatur:

1. Huttl TP, Obeidat FW, Parhofer KG et al. Zentralbl Chir 2009:134:24-31.

emanuel.fritschka@drv-nordbayern.de 